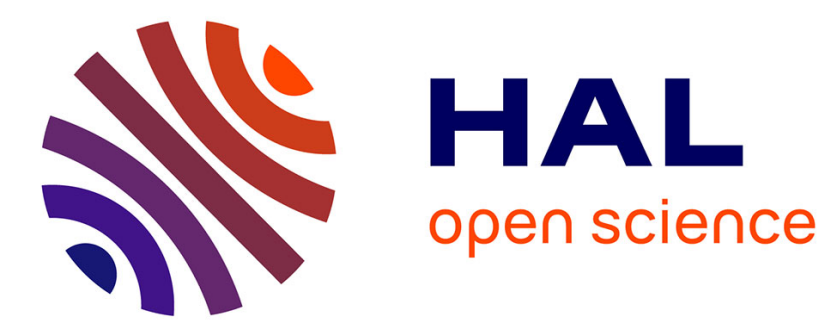

\title{
MRI temperature and velocity measurements in a fluid layer with heat transfer
}

\author{
Sébastien Leclerc, C. Métivier
}

\section{To cite this version:}

Sébastien Leclerc, C. Métivier. MRI temperature and velocity measurements in a fluid layer with heat transfer. Experiments in Fluids, 2018, 59 (2), 10.1007/s00348-018-2494-3 . hal-01693075

\section{HAL Id: hal-01693075 \\ https://hal.univ-lorraine.fr/hal-01693075}

Submitted on 25 Jan 2018

HAL is a multi-disciplinary open access archive for the deposit and dissemination of scientific research documents, whether they are published or not. The documents may come from teaching and research institutions in France or abroad, or from public or private research centers.
L'archive ouverte pluridisciplinaire HAL, est destinée au dépôt et à la diffusion de documents scientifiques de niveau recherche, publiés ou non, émanant des établissements d'enseignement et de recherche français ou étrangers, des laboratoires publics ou privés. 


\title{
MRI Temperature and Velocity measurements in a fluid layer with heat transfer
}

\author{
S. Leclerc • C. Métivier
}

Received : date / Accepted : date

Résumé Magnetic Resonance Thermometry (MRT) is an innovative technique which can provide $2 \mathrm{D}$ and 3D temperature measurements using Magnetic Resonance Imaging (MRI). Despite the powerful advantages of MRT, this technique is sparcely developed and used in the engineering sciences. In this paper, we investigate the possibility to measure temperatures with MRI in a fluid layer submitted to heat transfer. By imposing a vertical temperature gradient, we study the temperature fields in both conductive and convective regimes. The temperature fields are obtained by measuring the transverse relaxation time $T_{2}$ in glycerol, a Newtonian fluid. The MRT protocol is described in detail and the results are presented. We show that for a conductive regime, temperature measurements are in very good agreement with the theoretical profile. In the convective regime, when comparing the temperature and velocity fields obtained by MRI, we get an excellent agreement in terms of flow structure. Temperature uncertainties are found to be less than $1{ }^{\circ} \mathrm{C}$ for all our results.

Keywords Magnetic Resonance Thermometry MRT · MRI · Natural convection · Heat transfer

\section{Introduction}

An innovative technique to measure temperature is emerging in the engineering community, in particular in the field of fluid-thermal flows. This technique consists in measuring temperature by Magnetic Resonance Imaging (MRI) leading to a nonintrusive method which provides insightful results in two or three dimensions. In addition, images in transparent or opaque systems can be obtained by MRI, which is a huge

S. Leclerc

E-mail: sebastien.leclerc@univ-lorraine.fr

Tel. : $+33-372745260$

C. Métivier

E-mail: christel.metivier@univ-lorraine.fr

Tel. : $+33-372744279$

LEMTA (UMR 7563) - CNRS - Universtité de Lorraine 
advantage compared with other techniques such as thermochromic liquid crystals, heat sensitive paints, Laser-induced fluorescence (LIF), or Particle Imaging Thermometry (PIT) $[1,2]$. These latter techniques restrict the setup to transparent walls in order to make possible the flow visualizations. The MRT technique (Magnetic Resonance Thermometry) is widespread in the medical field (see reviews [3,4,5]). In this field, MRT is principally performed in vivo on systems at rest (without fluid motion). For instance, the MRT technique is used for thermal therapy which induces large temperature variations at very local areas and does not require a large resolution on the temperature values, leading to short time measurements.

Despite the powerful advantages of MRT, this technique is sparcely developed in the engineering sciences. To our knowledge, few studies, which measure temperature by MRI in the field of fluid mechanics and thermal sciences, have been published $[6,7$, 8]. The first study [6] presents qualitative results concerning the flow and heat transfer in a fluid layer of ethylene glycol submitted to a vertical temperature gradient for large values of temperature difference between the horizontal slabs (around $60^{\circ} \mathrm{C}$ ), and large values of the Rayleigh number. The studies $[7,8]$ investigate the flow of water with addition of a contrast agent. The latter is used to increase the accuracy of the measurements by increasing the signal to noise ratio and decreasing the experiments duration, in respectively a heat exchanger [7] and a fully turbulent jet mixing [8]. Validation of the studies $[7,8]$ is provided in [7] and [9].

The MRI Thermometry difficulties are associated to the indirect measurement technique which depends essentially on the fluid properties. Indeed, different MR parameters show a sensitivity to the temperature. Several NMR (Nuclear Magnetic Resonance) techniques may evaluate the thermo-dependency of a fluid $[3,4,5]$ :

(i) The material sensitivity to the temperature can be evaluated by considering the Proton Resonance Frequency (PRF). The temperature fields can be obtained by measuring the phase difference due to the resonance frequency variation occuring when the temperature is modified $[10,11,12,13]$. In this sense, this technique requires a reference phase obtained at a known temperature. This technique is the most common method used in the medical field. It has also been used in the temperature acquisition in the case of turbulent fluid flows including heat transfer $[7,8]$.

(ii) The molecular diffusion coefficient $D$ presents a linear variation with the temperature, as proposed in the Stokes-Einstein relationship : $D=\left(k_{b} T\right) /(6 \pi r \mu)$, where $k_{b}$ is the Boltzman's constant, $T$ is the absolute temperature, $r$ is the radius of the fluid particles submitted to a Brownian motion and $\mu$ is the fluid viscosity. This measurement can be performed by MRI [14] in a motionless system. When a motion occurs, what is measured is not a diffusion coefficient but a dispersion coefficient which combines the effects of the advective movement and the molecular diffusion, is measured. Therefore, this method is not indicated in fluid flows.

(iii) A third technique consists in measuring the longitudinal (or spin-lattice) relaxation time $T_{1}$ or the transverse (or spin-spin) relaxation time $T_{2}[15,16]$. The relaxation time measurements lead to absolute temperature values but require to know beforehand the correlation between the relaxation time and the temperature. This technique is not often used in medical applications because the correlation is usually unknown for the individual living tissues [4].

Depending of the chosen method, the temperature measurement is either absolute or relative. In the case where the measurement is relative, it involves a comparison with a reference obtained in similar experimental conditions and at relatively close times $[7$, $8]$. 
Our interest focuses on heat transfer in a specific fluid flow referred as the RayleighBénard Convection (RBC). It consists in a buoyancy-driven unstable configuration since a fluid layer is heated from below and cooled from above. The fluid motion occurs above a certain temperature difference $\Delta T_{c}$ between two horizontal walls. Below $\Delta T_{c}$, the thermal regime is conductive and the fluid is motionless. In the convective regime, the experiments last several days to several weeks because the convective intensity is progressively increased. As said above, the method (i) requires a priori a reference image at a known temperature. However, it is difficult to obtain a sufficiently stable magnetic field of the spectrometer to get accurate measurements in such time scale. As the results are sensitive to magnetic field, it is not possible to update the reference by acquiring a new reference image once the convective regime occurs in the frame of our experiments. On the other hand, as already discussed, the second method (ii) is not indicated in the presence of flow. Finally the technique (iii) which consists in measuring relaxation times seems to be the most relevant method for our configuration.

In this paper, we propose to investigate the MR Thermometry by measuring the relaxation times in a fluid layer submitted to heat transfer. For this purpose, we study first the conductive regime to validate the method as well as to obtain a physical calibration correlation between the temperature and the relaxation time for the tested fluid. Then, we extend this method to convective regimes. In addition to the MRT measurements, we also provide MR Velocity (MRV) measurements. MRV is a widespread technique and it has already been employed in the Rayleigh-Bénard configuration in [17]. The present paper focuses on the validity as well as the accuracy and uncertainties obtained using MRT.

The section hereafter aims at presenting the experimental materials, setup and MRI protocols. The results obtained for the different thermal regimes are provided in section 3. Concluding remarks are proposed in the last section.

\section{Methods}

\subsection{Fluid and setup}

The fluid used in our experiments is glycerol, a Newtonian fluid characterized by a constant viscosity $\mu=0.9 \mathrm{~Pa} . \mathrm{s}$ at $T=24{ }^{\circ} \mathrm{C}$. The viscosity variations with temperature have been measured using a TA Instruments rheometer AR2000 with a cone and plate geometry. The cone diameter is $6 \mathrm{~cm}$, its angle is $2^{\circ}$ with a truncation of $70 \mu \mathrm{m}$. The viscosity is depicted as a function of the temperature in figure 1 . The continuous line corresponds to the following model :

$$
\mu(T)=\mu\left(T_{r e f}\right) \exp \left(-K\left(T-T_{r e f}\right)\right)
$$

where $T_{\text {ref }}=24{ }^{\circ} \mathrm{C}$. Our rheological measurements lead to $K=0.0945\left({ }^{\circ} \mathrm{C}\right)^{-1}$.

The thermodynamics properties of glycerol can be found in $[18,19]$. The properties in the temperature range $[0,50]{ }^{\circ} \mathrm{C}$ are :

$$
\begin{aligned}
C_{p}(T)=4186.6 \times(54+0.15 T) \times 10^{-2} & {\left[\mathrm{~J} /\left(\mathrm{kg} .{ }^{\circ} \mathrm{C}\right)\right] } \\
\beta(T)=[47+0.2(T-20)] \times 10^{-5} & {\left[1 /{ }^{\circ} \mathrm{C}\right] }
\end{aligned}
$$




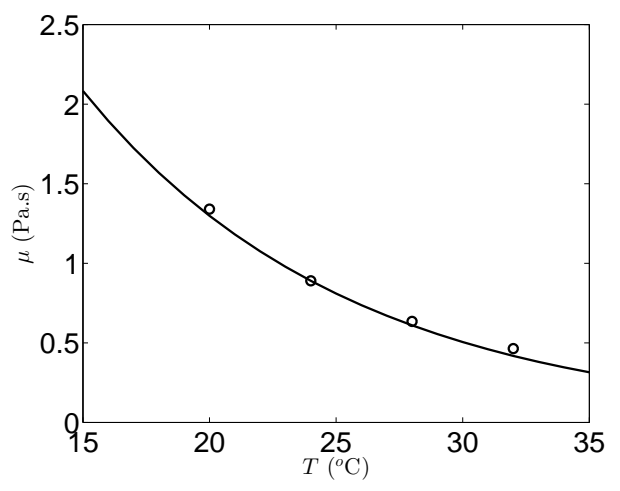

Figure 1: Glycerol viscosity as a function of temperature. Continuous line : $\mu\left(T_{\text {ref }}\right) \exp \left(-K\left(T-T_{\text {ref }}\right)\right)$, with $K=0.0945\left({ }^{\circ} \mathrm{C}\right)^{-1}$ and $T_{\text {ref }}=24{ }^{\circ} \mathrm{C}$

where $C_{p}$ is the thermal capacity and $\beta$ is the thermal expansion coefficient. At $T=$ $24{ }^{\circ} \mathrm{C}$, the thermal conductivity $\Lambda$ and the density $\rho$ are :

$$
\begin{array}{ll}
\Lambda\left(24^{\circ} \mathrm{C}\right)=0.28 & {\left[\mathrm{~W} /\left(\mathrm{m} .{ }^{\circ} \mathrm{C}\right)\right]} \\
\rho\left(24^{\circ} \mathrm{C}\right)=1259 & {\left[\mathrm{~kg} / \mathrm{m}^{3}\right]}
\end{array}
$$

The setup is the same as the one described in Darbouli et al. [17]. A cylindrical cavity is filled with glycerol, with an inner diameter $D=120 \mathrm{~mm}$ and a height $d=20$ $\mathrm{mm}$ as shown in figure 2 . The temperatures $T_{d}$ and $T_{u}$ are respectively imposed at the bottom and the top of the cavity via water flows. To this end, the horizontal sapphire plates of the cavity present a good thermal conductivity $\Lambda_{s}=35-40 \mathrm{~W} /(\mathrm{mK})$, while the lateral wall of the cylinder is made of polymethyl methacrylate (PMMA) involving thermal insulated conditions at this side. The temperatures of the water circulations are measured by thermocouples located at the inlet and the outlet. For all experiments, we observed weak temperature variations between the inlet and the outlet, i.e. around $0.1{ }^{\circ} \mathrm{C}$. All materials of the setup are non-metallic in order to not perturb the magnetic field of the spectrometer. For this reason, the thermocouples are located outside the MRI resonator.

Different thermal conditions can be obtained. First, by imposing similar temperature values at the upper and lower water circulations, the resulting isothermal regime in the glycerol layer is used in the calibration protocol §3.1. By imposing $T_{d}<T_{u}$, a conductive regime is obtained, it is characterized by a linear temperature stratification in the $O Z$ direction (see figure 2 for the definition of axes). Some experiments were conducted with these temperature conditions, results are given in $\S 3.2$. Finally, convective regime $(\S 3.3)$ can be obtained in the Rayleigh-Bénard situation, i.e. in the case where $T_{d}>T_{u}$ and when $\Delta T=T_{d}-T_{u}$ is large enough to imply a buoyancy-driven instability. The convection occurs when the buoyancy effects overcome the diffusive effects due to the viscosity and the thermal diffusivity $\kappa$. The onset of convection in this situation is governed by the Rayleigh number defined by $R a=\frac{\rho g \beta \Delta T d^{3}}{\mu \kappa}$, where $g$ is the gravitational acceleration. In the case of Newtonian fluids and an infinite aspect ratio $A=D / d$, the criticality is $R a_{c}=1708$ and the patterns are parallel rolls [20, 


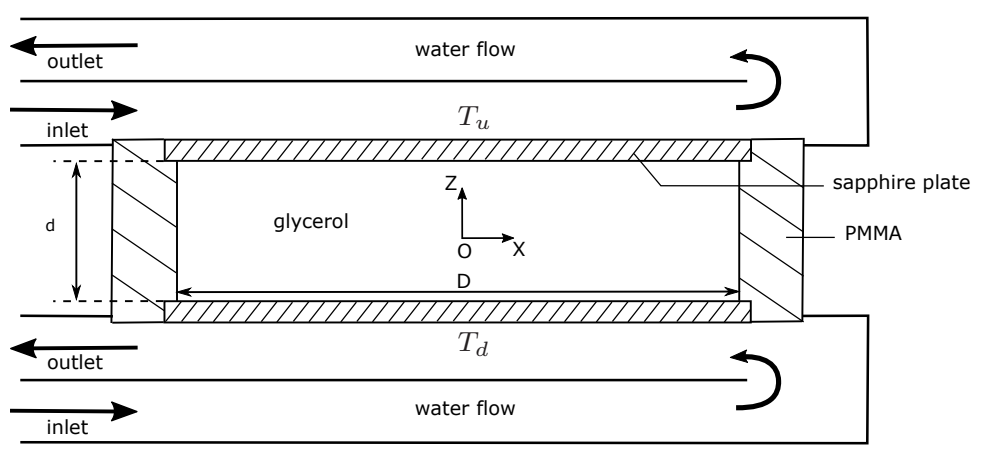

Figure 2: Scheme of the setup

21]. When the aspect ratio $A$ is decreased, $R a_{c}$ increases and lead to $R a_{c}=1725$ for $A=6[22,23]$.

Except in isothermal conditions, the mean temperature $T_{m}=T_{r e f}$ of the two horizontal slabs is always set in our experiments to $24{ }^{\circ} \mathrm{C}$. Furthermore, in the conductive regime, the temperatures $T_{d}$ and $T_{u}$ were imposed and held for about 2 hours to reach a steady state. In the Rayleigh-Bénard situation $\left(T_{d}>T_{u}\right)$, each experimental step is characterized by an increase in $\Delta T$ of a value $\delta T$. When approaching the transition between conductive to convective regimes and above the criticality, we wait 8 hours at each $\Delta T$ steps.

\subsection{NMR basics - relaxation time}

Relaxation in NMR is the process by which magnetization, after a perturbation such as a radiofrequency pulse, goes back to its equilibrium state. To take into account this effect, two relaxation times are used : $T_{1}$ for the longitudinal component of the magnetization and $T_{2}$ for the transverse one. The relaxation mechanisms are described using Bloch equations :

$$
\begin{aligned}
& \frac{d M_{x_{i}}}{d y}=\gamma\left(\mathbf{M} \wedge \mathbf{B}_{0}\right)_{x_{i}}-\frac{M_{x_{i}}}{T_{2}}, \quad \text { with } i=1 \text { or } 2 \\
& \frac{d M_{x_{3}}}{d y}=\gamma\left(\mathbf{M} \wedge \mathbf{B}_{0}\right)_{x_{3}}-\frac{M_{0}-M_{x_{3}}}{T_{1}}
\end{aligned}
$$

with $\gamma$ the gyromagnetic ratio of the considered spin, $\mathbf{M}$ the magnetization vector and $\mathbf{B}_{0}$ the static magnetic field vector which is along $x_{3}$. The longitudinal relaxation (or spin-lattice relaxation) corresponds to a reconstruction of the magnetization vector along the static magnetic field axis ( $x_{3}$ axis). On the contrary, transverse relaxation (or spin-spin relaxation) is a disappearance of the magnetization vector in the plane transverse to the magnetic field $\left(x_{1}, x_{2}\right)$ plane. 


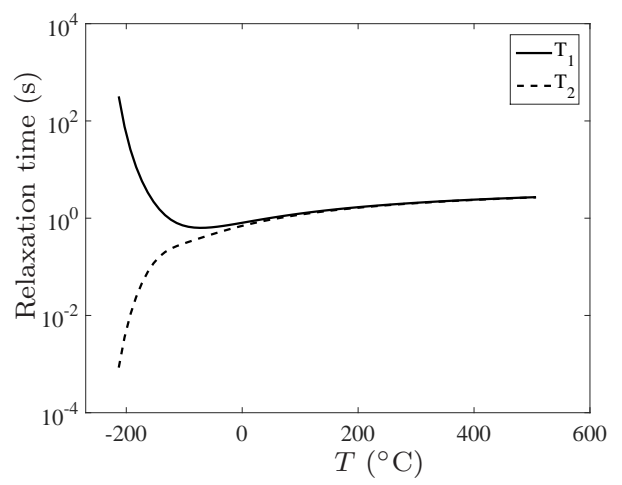

Figure 3: Variations of the relaxation times $T_{1}$ and $T_{2}$ as a function of temperature using the BPP model for typical values of $r$ and $\tau_{c}$ corresponding to small molecules such as water.

A good approximation of the temperature dependence of $T_{1}$ and $T_{2}$ can be obtained using the Bloembergen Purcell Pound (BPP) model [15] for a two spins system :

$$
\begin{aligned}
\frac{1}{T_{1}} & =\left(\frac{\mu_{0}}{4 \pi}\right)^{2} \frac{3}{10 r^{6}} \gamma^{4} \hbar\left[\frac{\tau_{c}}{1+\omega_{0}^{2} \tau_{c}^{2}}+\frac{4 \tau_{c}}{1+4 \omega_{0}^{2} \tau_{c}^{2}}\right] \\
\frac{1}{T_{2}} & =\left(\frac{\mu_{0}}{4 \pi}\right)^{2} \frac{3}{20 r^{6}} \gamma^{4} \hbar\left[3 \tau_{c}+\frac{5 \tau_{c}}{1+\omega_{0}^{2} \tau_{c}^{2}}+\frac{2 \tau_{c}}{1+4 \omega_{0}^{2} \tau_{c}^{2}}\right] \\
\tau_{c} & =\tau_{c 0} \exp \left(\frac{\Delta E}{k_{b} T}\right)
\end{aligned}
$$

where $r$ is the inter-spin distance, $\tau_{c}$ is the correlation time, representative of the molecule mobility, $\omega_{0}=\gamma\left|\mathbf{B}_{0}\right|, \mu_{0}$ the vacuum magnetic permeability, $\hbar=h /(2 \pi)$ the reduced Planck constant. Figure 3 shows the variations of $T_{1}$ and $T_{2}$ with the temperature for typical values of $r$ and $\tau_{c}$ corresponding to small molecules such as water. The $T_{1}$ curve reaches a minimum value whose position depends on the correlation time $\tau_{c}$ and therefore the molecular mobility.

\subsection{MRI protocols}

MRI experiments were performed on a Bruker Avance Biospec 24/40 spectrometer characterized by a $2.4 \mathrm{~T}$ horizontal magnetic field corresponding to a proton resonance frequency of $100.3 \mathrm{MHz}$. The spectrometer is equipped with a $200 \mathrm{~mm}$ inner diameter gradient coil delivering a $200 \mathrm{mT} / \mathrm{m}$ gradient strength. Our experimental setup is positioned in a $160 \mathrm{~mm}$ diameter quadrature resonator provided by Doty.

\section{MR Thermometry}

$T_{1}$ images are obtained by acquiring different spin echo images [24] with different repetition time $T_{R}$. The value of $T_{R}$ has been varied between $30 \mathrm{~ms}$ and $750 \mathrm{~ms}$, the echo time $T_{E}$ has been set to $11 \mathrm{~ms}$. The signal for each image increases with $T_{R}$, as the spins have more time to return to equilibrium and therefore the magnetization vector 


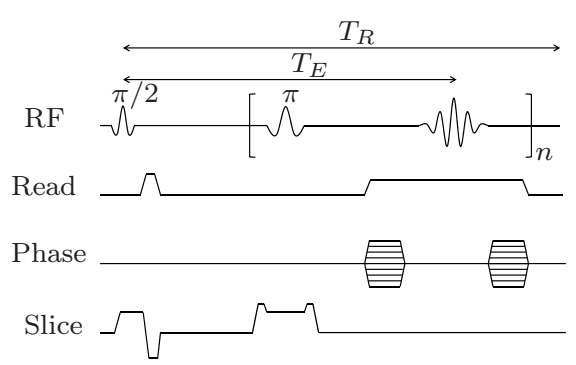

(a)

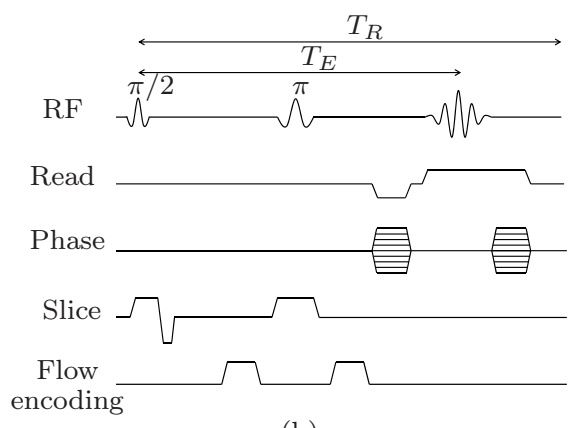

(b)

FIGURE 4: MRI sequences for the (a) relaxation time measurements and (b) the velocity measurements. In sequence (a), $n$ is the number of consecutive echoes acquired $(n=1$ for $T_{1}$ and $n=8$ for $T_{2}$ )

reconstructs along the static magnetic field axis. The relaxation map is then obtained using the following equation applied for each pixel :

$$
S_{\text {pixel }} \propto\left(1-\exp \left(\frac{-T_{R}}{T_{1}}\right)\right) .
$$

$T_{2}$ images are obtained using a multi-spin echo sequence [24] (see Fig. 4(a)) with 8 consecutives echoes. The images are averaged 16 times in order to improve the signal to noise ratio. The echo time $T_{E}$ is set to $12 \mathrm{~ms}$ with a repetition time $T_{R}=500 \mathrm{~ms}$. The signal for each image decreases with each echo. Finally, the relaxation map is then reconstructed pixel by pixel using the following equation :

$$
S_{\text {pixel }} \propto\left(\exp \left(\frac{-T_{E}}{T_{2}}\right)\right) .
$$

\section{MR Velocity}

Velocity images are obtained using a protocol detailed in [17]. This protocol uses a classical spin-echo imaging sequence with additional bipolar gradient pulses in order to encode the velocity [24]. The sequence is depicted in Fig. 4(b). Two images are acquired with different gradient amplitude $(0$ and $180 \mathrm{mT} / \mathrm{m})$ and the difference of the two images gives the velocity image. Spin echo sequence is used because it is immuned to the chemical shift effect arising from the temperature difference, as verified in section 3.2. The following parameters have been used : the gradient pulses duration $\delta=4 \mathrm{~ms}$, the interval between two gradient pulses $\Delta=12 \mathrm{~ms}, T_{E}=28 \mathrm{~ms}$ and $T_{R}=500 \mathrm{~ms}$. The images are averaged 4 times.

The velocity resolution is the order of $10^{-5} \mathrm{~m} / \mathrm{s}$ [25]. Considering our experimental setup, we obtain a velocity uncertainty of 5 times the resolution [17]. It means that we cannot measure velocity magnitudes below the value $5 \cdot 10^{-5} \mathrm{~m} / \mathrm{s}$.

For both MRT and MRV protocols, the slice thickness is $2 \mathrm{~mm}$ for every images. For horizontal images, the field of view FOV is $12 \mathrm{~cm} \times 12 \mathrm{~cm}$, the image matrix is $64 \times 64$ pixels, leading to a spatial resolution of $1875 \times 1875 \mu \mathrm{m}$. For vertical images, 
the FOV is $2.5 \mathrm{~cm} \times 12 \mathrm{~cm}$ with an image matrix of $64 \times 64$ pixels. It is worth noting some dephasing noise coming from the turbulent flow in the water channels at the top and bottom of the test section. In order to avoid the propagation of this noise in the whole resulting images, the acquisition of the vertical $X O Z$ planes is done by setting the phase gradient horizontal (X direction).

\section{Results}

All MRI measurements, i.e. velocity and relaxation times measurements, have been performed in 2D vertical $(X O Z)$ and horizontal $(X O Y)$ cross-sections.

\subsection{Calibration}

The calibration is obtained by imposing isothermal conditions in the fluid layer. It aims at defining a correlation between the relaxation times $T_{1}$ or $T_{2}$ with the temperature. Results are displayed in figure 5.

Concerning the longitudinal relaxation time $T_{1}$, our results are in agreement with results given by Kruk et al. [26]. We observe that two values of temperature are possible for a given $T_{1}$. Because it is difficult to discriminate one temperature value, the measurement of $T_{1}$ is not appropriate to obtain the real temperature field. The high microscopic viscosity of glycerol and therefore its long correlation time $\tau_{c}$ is such as the minimal value of $T_{1}$ is located in the range of our experimental temperatures. In the case of small molecules (water for instance), this minimum would occur at a smaller temperature range. In this sense, $T_{1}$ could be used for other fluids provided that the minimum is not in the temperature range of interest. Concerning the transverse relaxation time $T_{2}$, the results can be fitted by a bijective function in the range of tested temperatures (see continuous line in figure $5(\mathrm{~b})$ ) since for a given value of $T_{2}$ corresponds only one value of temperature. In the following, we only present the temperature fields obtained from $T_{2}$ measurements.

\subsection{Conductive regime}

As a first validation of the MRI thermometry, we have considered a simple case : a pure conductive regime in a fluid layer at rest. We present the results corresponding to $\Delta T=-9.7^{\circ} \mathrm{C}$ and $\Delta T=-19.5^{\circ} \mathrm{C}$ in figure 6 . For both $\Delta T$ values, a vertical stratification of the temperature field in the $(X O Z)$ plane is observed. It corresponds effectively to a linear temperature profile along the vertical coordinate as shown in figure 6 for both cases $\Delta T=-9.7^{\circ} \mathrm{C}$ and $\Delta T=-19.5^{\circ} \mathrm{C}$. The uncertainty is found to be less than $1{ }^{\circ} \mathrm{C}$. The MRT technique provides very good results for motionless fluids.

It can be noticed that a small deviation occurs in the temperature field when approaching the side wall $(X= \pm 0.06 \mathrm{~m}$ ). This deviation is due to the inhomogeneity of the radiofrequency field at boundaries of a large object, i.e. when the dimensions of the object (setup) are of the same order as the resonator ones. This leads to inaccurate pulses calibrations near the side wall of the setup, and thus to imperfect $\pi$ pulses in 


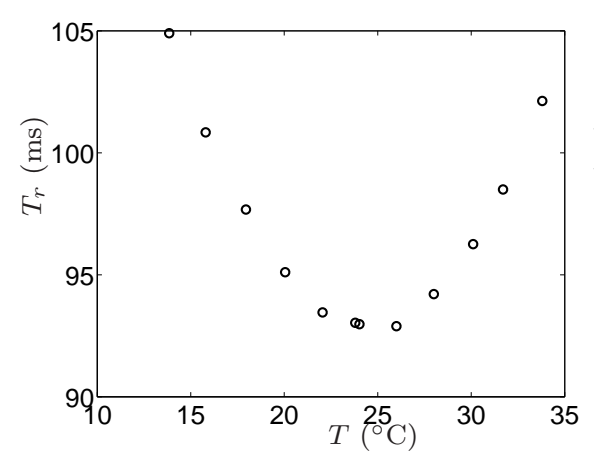

(a) $T_{r}=T_{1}$

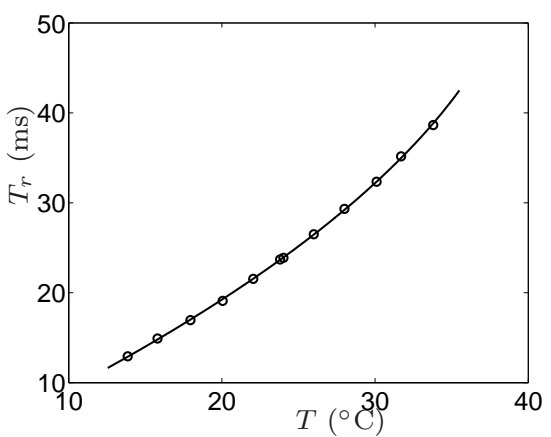

(b) $T_{r}=T_{2}$

Figure 5: Relaxation times as function of temperature in isothermal conditions (a) Longitudinal relaxation time $T_{1}$ and (b) Transverse relaxation time $T_{2}$. Continuous line : second order polynomial fit.
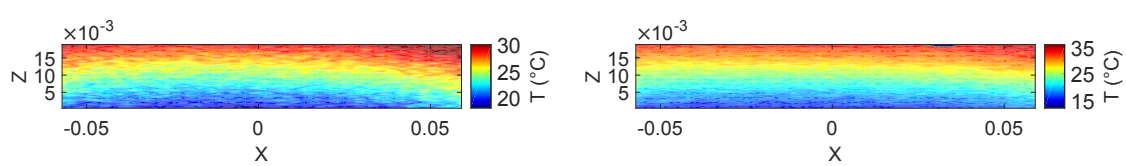

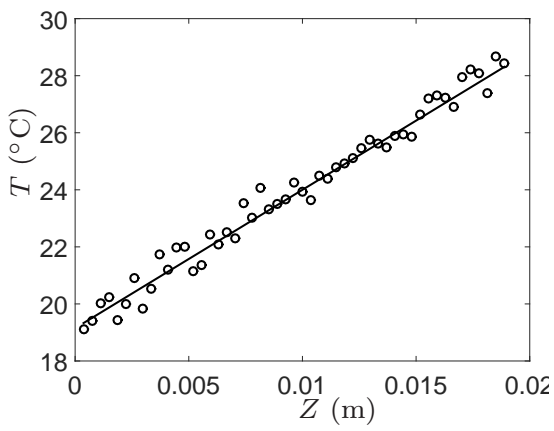

(a) $\Delta T=-9.7^{\circ} \mathrm{C}$

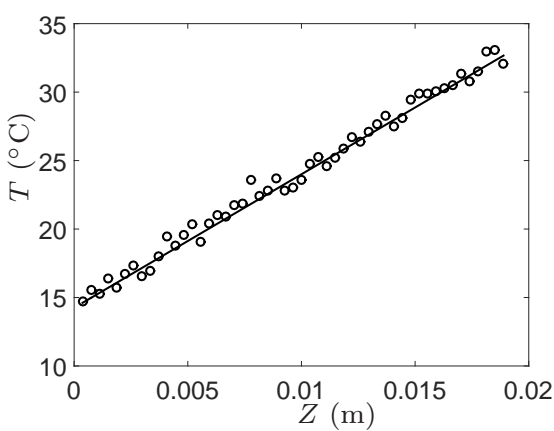

(b) $\Delta T=-19.5^{\circ} \mathrm{C}$

Figure 6: Temperature field (upside) of a conductive regime in the $X O Z$ plane and temperature profile at $X=0$ (downside) along the vertical coordinate $Z$ when (a) $\Delta T=-9.7^{\circ} \mathrm{C}$ and (b) $\Delta T=-19.5^{\circ} \mathrm{C}$. Continuous lines : Theoretical conductive temperature profile.

the spin echoes. The direct consequence is a slight error in the $T_{2}$ measurements in this region.

In velocity images, we have checked that dephasing caused by temperature does not occur due to the bipolar gradients. For this purpose, the dephasing has been measured in the case $\Delta T=-19.5^{\circ} \mathrm{C}$. In the range of our experimental parameters, we did not observe any significant dephasing caused by temperature variations in the velocity sequences. 
3.3 Convective regime

Two sets of experiments (EXPE1 and EXPE2) have been performed at different times in order to check the reproducibility of the results. The protocols EXPE1 and EXPE2 differ slightly : $\delta T$ is set to $1^{\circ} \mathrm{C}$ for EXPE1 and $\delta T=0.5^{\circ} \mathrm{C}$ for EXPE2. In our experiments, the onset of convection occurs between $R a=1820$ and $R a=1990$. Compared with the literature for Newtonian fluids which gives the critical Rayleigh number $R a_{c}=1725$, the value obtained in our experiments is within $10 \%$ error. The error is also due to the relatively large value of $\delta T$. At the onset, the convective pattern consists in unclear cells. Within the experimental resolution at very low velocity values, it is difficult to identify if these cells correspond to hexagons, squares or any other pattern. By increasing the Rayleigh number, rolls appear at the expense of cells which disappear as shown in the horizontal plane in figure 7 .

The effect of an increase in $R a$ values is an increase in the magnitude of the vertical component of the velocity $V_{Z}$ as well as a change in the rolls direction (EXPE2) or only a distortion of rolls (EXPE1). Nevertheless, both experiments lead to stable rolls until $R a=7800$. On the other hand, the results between velocity and temperature fields agree qualitatively for each experiment. Indeed, the shape and size of the pattern are similar for a given $R a$. In addition, the colder regions (light blue for the temperature fields) correspond effectively to down-flow fluid regions, i.e. regions where $V_{Z}<0$ (blue regions for the velocity fields) and the reciprocal situation is also observed (see red regions for both the velocity and temperature fields). The comparison with RBC results in glycerol proposed by Stasiek [27] also provides a qualitative validation of our results. Indeed, in this article, stream lines and only one isotherm visualisations (see Fig. 18a in [27]), obtained by using chiral-nematic liquid crystal in a fluid layer of glycerol, are provided for $R a=12000$. We obtain similar type of isotherms shape than the one given by Stasiek [27].

Similarly, our results are displayed in the vertical (XOZ) section of the cavity as shown in figure 8. In this plane, it is possible to superimpose to the temperature field (in color) the velocity vectors $\mathbf{V}=V_{X} \mathbf{e}_{X}+V_{Z} \mathbf{e}_{Z}$ (arrows). Again, results agree qualitatively. Furthermore, the temperature values obtained by MRT are realistic with respect to the values imposed at the walls. For instance, in Fig. 8, we impose walls temperature $T_{d}=31.1^{\circ} \mathrm{C}$ and $T_{u}=17.4^{\circ} \mathrm{C}$. Experimental temperature values obtained via MRT lead to $T_{d}^{M R T}=31.9^{\circ} \mathrm{C}$ and $T_{u}^{M R T}=16.4^{\circ} \mathrm{C}$ around $X=0$, where $T_{d}^{M R T}$ and $T_{u}^{M R T}$ correspond to a mean value obtained from 7 pixels centered around $X=0$. As previously, results highlight around $1{ }^{\circ} \mathrm{C}$ uncertainties close to the horizontal walls.

Finally, a video is proposed as the Online Resource 1. Each image of this video corresponds to the temperature field in the vertical $(X O Z)$ plane. It has been obtained at a given $\Delta T$ value during EXPE1 and each image has been acquired after waiting 8 hours at each step. By considering this video, the onset and the increase of the thermal plumes can be observed at each step of the experiment.

\section{Concluding remarks}

In conclusion, the MRI Thermometry provides a powerful, non-invasive and quantitative flow visualization method, capable of measuring temperature and velocity fields in transparent and opaque systems, in two dimensions and three dimensions. 


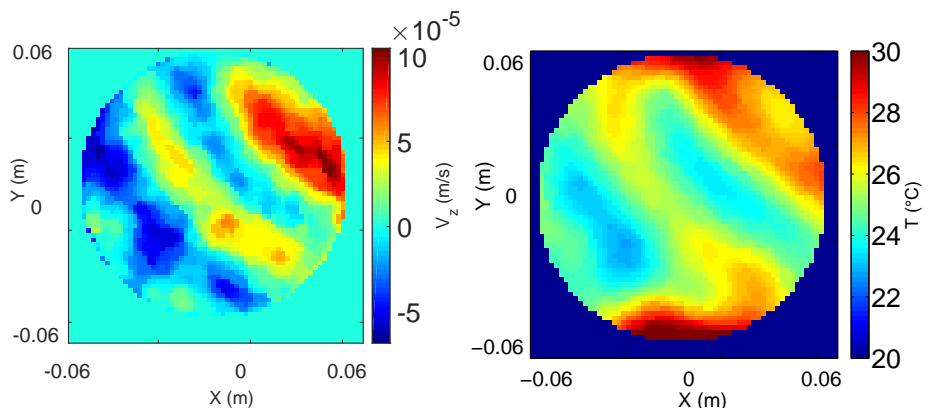

(a) $\Delta T=4.7, R a=2680$

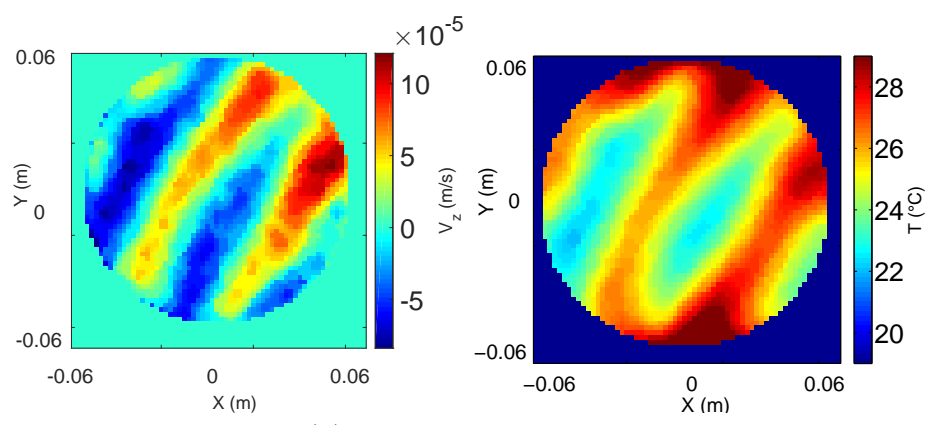

(b) $\Delta T=7.2, R a=4095$

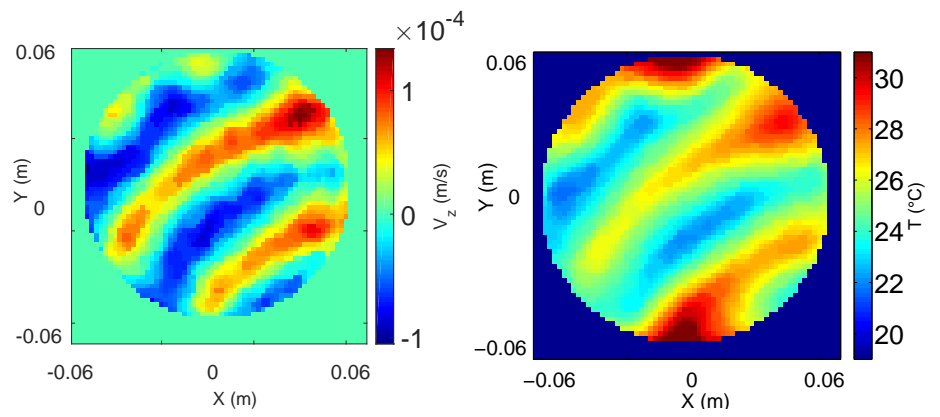

(c) $\Delta T=9.1, R a=5170$

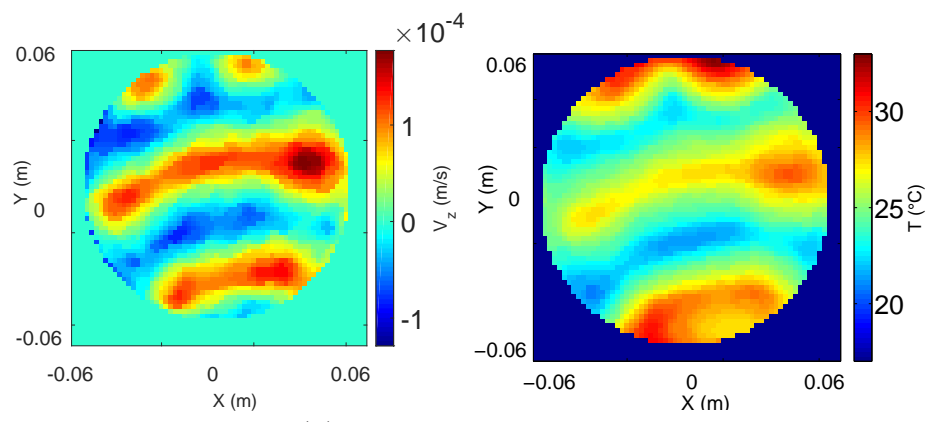

(d) $\Delta T=11.5, R a=6550$

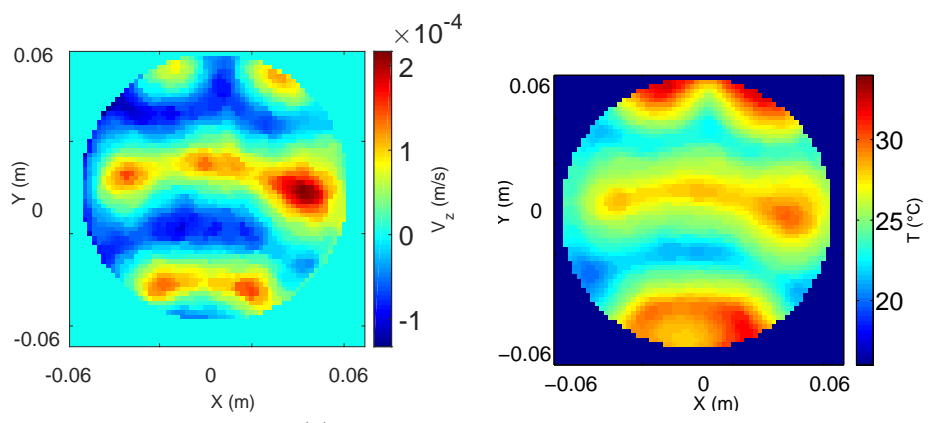

(e) $\Delta T=13.5, R a=7670$

Figure 7: Velocity (left) and Temperature (right) fields in the midle horizontal (XOY) plane of the cavity for different $\Delta T$ and $R a$ values - Results from EXPE2 


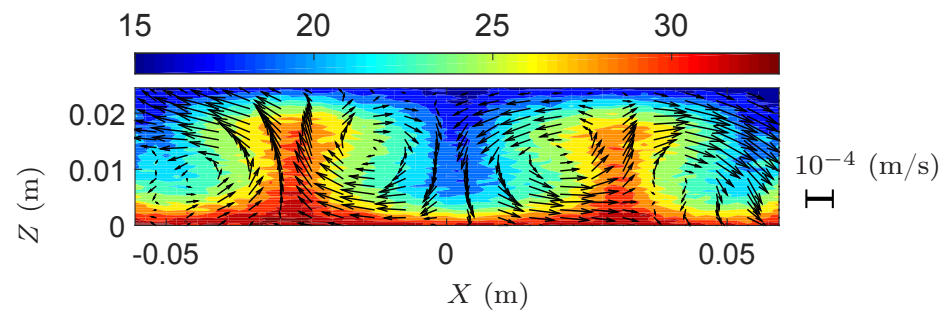

FiguRE 8: Superimposition of the velocity vectors on the temperature field for $\Delta T=$ 13.7, $R a=7795$ - Results from EXPE1. The small line on the right side gives a reference for the velocity magnitudes.

In the glycerol case, temperature fields have been obtained via the sensitivity of the transverse relaxation time $T_{2}$ with temperature. The $T_{2}$ sensitivity of glycerol is sufficiently large to lead to a good results accuracy. The temperature uncertainties are found to be less than $1{ }^{\circ} \mathrm{C}$ for all our results. The uncertainties can be larger in the regions where the radiofrequency field becomes inhomogeneous which is the case at the boundaries of a large setup.

In addition, in the convective regime, when comparing the temperature and velocity fields obtained by MRI, we get an excellent agreement in terms of flow structure.

A perspective to this work is an extension of the MRT technique to other fluids, for example fluids aqueous based such as polymer in water solutions characterized by a non-Newtonian behavior. Different difficulties may appear since the values of $T_{1}$ and $T_{2}$ are two orders of magnitude larger that in glycerol. This involves a longer data acquisition time and possibly a decrease in the signal to noise ratio. To avoid these difficulties, some contrast agents can be added. However, it is not always compatible with the use of polymers because it can change their physico-chemical properties and therefore their rheological properties. The improvement of the accuracy measurements is another perspective in a close future in order to be able to evaluate heat transfer.

\section{Références}

1. P.R. Childs, J.R. Greenwood, C.A. Long, Review of temperature measurement, Rev. Sci. Instum., 71, 2959-2978 (2000)

2. D. Dabiri, Digital particle image thermometry/velocimetry : a review, Exp. Fluids, 46, 191-241 (2009)

3. B. Quesson, J.A. de Zwart and C. T.W. Moonen, Magnetic resonance temperature imaging for guidance of thermotherapy, J. Magn. Reson. Imaging, 12, 525-533 (2000)

4. V. Rieke, K.B. Pauly, MR Thermometry, J. Magn. Reson. Imaging 27, 376-390 (2008)

5. R. Turner et M. Streicher, Measuring temperature using MRI : a powerful and versatile technique, Magn. Reson. Matter Phy., 25, 1-3 (2012)

6. M. Weber et R. Kimmich, Rayleigh-Bénard percolation transition in thermal convection in porous media : Computational fluid dynamics, NMR velocity mapping, NMR temperature mapping, Physical Review E, 66, 056301 1-056301 13 (2002)

7. W.B. Buchenberg, F. Wassermann, S. Grundmann, B. Jung, R. Simpson, Acquisition of 3D Temperature Distributions in Fluid Flow Using Proton Resonance Frequency Thermometry, Magn. Reson. Med. 76, 145-155 (2016) 
8. E.T. Williams, J.R. Spirnak, M.C. Samland, B.G. Tremont, A.L. McQuirter, C.M. Verhulst, B.P. Van Poppel, M. J. Benson, C.J. Elkins, L.S. Burton, J.K. Eaton, Magnetic Resonance Thermometry experimental setup : a portable heat transfer experiment, Proceedings of the ASMA 2016 IMECE, Nov. 11-17 Phoenix, Arizona USA

9. J.R. Spirnak, M.C. Samland, B.G. Tremont, A.L. McQuirter, E.T. Williams, M. J. Benson, B.P. Van Poppel, C.M. Verhulst, C.J. Elkins, L.S. Burton, J.K. Eaton, M. Owkes, Validation of Magnetic Resonance Thermometry through experimental and computational approaches, AIAA 2016-4741, AIAA Propulsion and Energy forum, July 25-27, 2016 Salt Lake City, UT, 52nd AIAA/SAE :ASEE Joint Propulsion Conference.

10. J.C. Hindman, Proton resonance shift of water in gas and liquid states, J. Chem. Phys., 44, 4582-4592 (1966)

11. Y. Ishihara, A. Calderon, H. Watanabe, A precise and fast temperature mapping using water proton chemical shift, Magn. Reson. Med., 34, 814-823 (1995)

12. J. de Poorter, C. de Wagter, Y. de Deene, C. Thomsen F. Stahlberg, E. Achten, Noninvasive MRI thermometry with the proton resonance frequency (PRF) method : in vivo results in human muscle, Magn. Reson. Med., 33, 74-81 (1995)

13. E.M. Shapiro, A. Borthakur, M.J. Shapiro, R. Reddy, J.S. Leigh, Fast MRI of RF heating via phase difference mapping, Magn. Reson. Med., 47, 492-498 (2002)

14. J. Delannoy, Ching-Nien Chen, R. Turner, R.L. Levin, D. Le Bihan, Noninvasive temperature imaging using diffusion MRI, Magn. Reson. Med. 19, 333-339 (1991)

15. N. Bloembergen, E.M. Purcell, R.V. Pound, Relaxation effects in nuclear magnetic resonance absorption, Phys. Rev., 73 (7), 679-712 (1948)

16. D.L. Parker, Applications of NMR imaging in hyperthermia : an evaluation of the potential for localized tissue heating and noninvasive temperature monitoring, IEEE Trans. Biomed. Eng. 31, 161-167 (1984)

17. M. Darbouli, C. Métivier, S. Leclerc et al, Natural convection in shear-thinning fluids : Experimental investigations by MRI, Int. J. Heat Mass Transfer 95, 742-754 (2016)

18. A.A. Newman, Glycerol, CRC Press (1968)

19. K.C. Stengel, D.S. Oliver, J.R. Booker, Onset of convection in a variable-viscosity fluid, J. Fluid Mech. 120, 411-431 (1982)

20. L. Rayleigh, On convective currents in a horizontal layer of fluid when the higher temperature is on the under side, Phil. Mag. 32, 529-546 (1916)

21. A. Schluter, D. Lortz, F. Busse, On the stability of steady finite amplitude convection, J. Fluid Mech. 23, 129-144 (1965)

22. J.C. Buell, I. Catton, The effect of wall conduction on the stability of a fluid in a right circular cylinder heated from below, ASME J. Heat Transfer 105 (2), 255-260 (1983)

23. F. Hébert, R. Hufschmid, J. Scheel, G. Ahlers, Onset of Rayleigh-Bénard convection in cylindrical containers, Phys. Rev. E 81, 046318 (2010)

24. M.A. Bernstein, K.F. King, X.J. Zhou, Handbook of MRI pulse sequences, Elsevier (2004)

25. W. Salameh, Imagerie par résonance magnétique nucléaire pour la vélocimétrie d'un écoulement en milieu poreux (Ph.D. thesis), Institut National Polytechnique de Lorraine (2011)

26. D. Kruk, A. Korpala, E. Rossler, K.A. Earle, W. Medyfki, J. Moscicki, ${ }^{1}$ H NMR relaxation in glycerol solutions of nitrodide radicals : Effects of translational and rotational dynamics, J. Chem. Phys. 136, 114504 1-8 (2012)

27. J. Stasiek, Thermochromic liquid crystals and true colour image processing in heat transfer and fluid-flow research, Heat and Mass Transfer 33, 27-39 (1997) 